\title{
Una plataforma para la docencia en Economía
}

\author{
Penélope Hernández Rojas \\ Profesora titular del Departament d'Anàlisi Econòmica de la \\ Universitat de València \\ Penelope.Hernandez@uv.es
}

\author{
José Manuel Pastor \\ Profesor titular del Departament d'Anàlisi Econòmica de la \\ Universitat de València
}

Jose.M.Pastor@uv.es

|Fecha presentación: 04/05/2009 | Aceptación: 12/05/2009 |Publicación: 06/07/2009

\section{Introducción}

La actual sociedad del conocimiento otorga a las universidades un papel fundamental a la vez que singular. Las universidades son las únicas instituciones que participan en todos los procesos relacionados con el mismo en su actividad cotidiana: 1) generan conocimiento a través de la investigación; 2) lo transmiten por medio de la educación de graduados y la formación de investigadores; y 3) explotan sus resultados gracias a la cooperación industrial y el aprovechamiento de las ventajas tecnológicas con empresas e instituciones de su entorno. Como resultado del desempeño de estas funciones surge una estrecha y fructífera vinculación entre la universidad y la sociedad a la que pertenece que, sin duda, resulta mutuamente beneficiosa para las dos partes ${ }^{2}$.

El proceso de Bolonia supone una revolución fundamental en la segunda de las funciones desempeñadas por las universidades: la función de trasmisión y difusión del conocimiento. Aunque el proceso de Bolonia no erosiona en modo alguno esta función de las universidades sí que supone una profunda transformación en el modo en el que las mismas y, concretamente sus profesores, desarrollan esta función.

Bolonia va más allá de un mero cambio de nomenclaturas o de programas: es una auténtica evolución en las enseñanzas superiores europeas, en la que el docente representa un papel protagonista. La transformación es de tal calibre que podríamos calificarla de metamorfosis. Tanto es así que muchos profesores universitarios no han asimilado en su totalidad la dimensión del cambio que se les avecina. Ante esta situación los docentes tienen dos estrategias: permanecer fieles a los métodos docentes tradicionales o seguir una estrategia proactiva, que sepa sacar el máximo partido de las nuevas metodologías docentes.

La transformación que supone el proceso de Bolonia no puede, ni debe, realizarse de manera inmediata. El cambio de la cultura docente de los profesores y, concretamente, en los modos de concebir y desarrollar la metodología que rige los procesos de enseñanza-aprendizaje es un proceso largo que no todos los docentes pueden (o desean) realizar con igual celeridad.

La transformación es importante, pero no debe asustar ni a las instituciones ni a sus docentes. Las universidades españolas y sus profesores tienen experiencia haciendo frente a distintas transformaciones en el pasado reciente, como el creciente número de estudiantes y los episodios de masificación, el proceso de descentralización, los cambios en el marco normativo, la ampliación y diversificación de la oferta de titulaciones, etc. Por eso se conocen las dificultades de adaptación y las rigideces que padecen algunas de sus estructuras. Por ello, partiendo de la experiencia del pasado, y dado que los retos que ahora se plantean no son menores en magnitud, debemos tener presente que las numerosas adaptaciones que se tienen que abordar requerirán por parte de las instituciones una adecuada programación para anticiparse, acierto en la definición de la estrategia, aumento de la flexibilidad de sus organizaciones, apoyo organizativo y financiero a las iniciativas docentes innovadoras y, por supuesto, compromiso, esfuerzo y un comportamiento proactivo por parte de los docentes.

Para movilizar todos esos recursos financieros, materiales y humanos será imprescindible que la comunidad universitaria comparta la visión de que la construcción de la sociedad del conocimiento requiere colocar la calidad de la docencia en un lugar muy destacado, que nunca debió per-

\footnotetext{
${ }^{1}$ Los autores agradecen el apoyo financiero del Ministerio de Ciencia e Innovación de los proyectos SEJ2007-66581 y EC02008-03813 respectivamente. 2. Como se puede ver en Pérez et al. (2006) y Michavila et al. (2007)
} 
der. Para ello es necesario definir criterios para evaluar la calidad acordes con el contexto social y tecnológico actual, difundirlos adecuadamente y reconocer la excelencia en este ámbito, otorgándole la misma relevancia que merece la excelencia investigadora. Éste es un reto adicional a los que acabamos de enunciar, que se sitúa en el interior de las universidades, pero no por ello es menos exigente.

En este sentido la Universitat de València a través de su Vicerectorat de Convergència Europea i Qualitat ha mostrado una notable sensibilidad a los retos antes mencionados. En particular, dicho vicerrectorado se ha involucrado de manera activa en la mejora y efectividad del aprendizaje a través del desarrollo de materiales específicos, de la utilización de la herramienta del Aula Virtual, que permite un contacto personal con los alumnos, y por supuesto en la mejora para la adaptación al Espacio Europeo de Educación Superior. Son destacables las ayudas propuestas en su programa específico de innovación, y los cursos especializados que permiten al cuerpo docente conocer las nuevas tecnologías para mejorar sus actividades. Además, es de notar el apoyo que se brinda a nuevos proyectos innovadores. Uno de ellos es la iniciativa, nacida el pasado año por parte de un grupo de profesores de Economía de varias universidades españolas, y que cuenta con el apoyo de este vicerrectorado. La colaboración entre las universidades de Granada, Murcia y Valencia dio como fruto la formación de una plataforma docente donde los profesores en estas áreas comparten y mejoran sus materiales y experiencias docentes.

2. La necesidad de una plataforma docente en Economía La constatación de los importantes cambios en relación a la docencia que implica el proceso de Bolonia, y la soledad en la que tales transformaciones deben llevarse a cabo por parte del profesorado, impulsó a un grupo de profesores de diversas universidades públicas españolas a plantear la necesidad de que los docentes intercambiaran opiniones y experiencias en el ámbito docente, al igual que se hace en el ámbito de investigación.

Concretamente, tres fueron los detonantes que promovieron el surgimiento de esta iniciativa:

a) La necesidad de adaptar la docencia a nuevas orientaciones y formatos como el sistema de créditos ECTS, en el que se basa la enseñanza dentro del Espacio Europeo de Educación Superior.

b) Los posibles incentivos a la buena praxis docente.

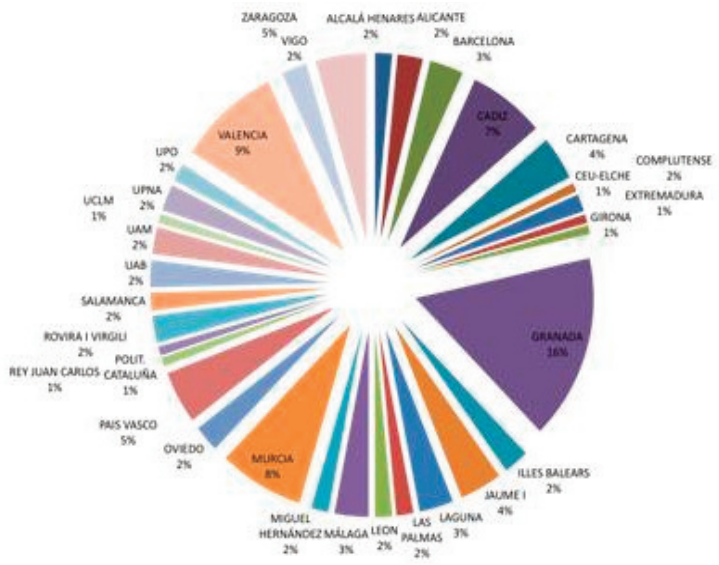

Gráfico 1. Distribución porcentual de la asistencia por universidades c) La necesidad de que estos cambios no se desarrollen de modo endógeno entre los profesores de cada materia sino intercambiando experiencias con colegas de otras universidades.

Lo anterior implica que se haga imprescindible que el material docente cubra las principales exigencias del marco europeo. Este material debe adaptarse al menor peso de las clases presenciales, por lo que un adecuado diseño contribuirá a agilizar la metodología docente de las clases magistrales, complementándolas con clases prácticas, seminarios, trabajos en grupo, etc.

Asimismo, nuestras universidades, a través de diversas iniciativas (contratos-programas, tramos autonómicos y nuevo estatuto del PDI), se están mostrando más proclives a incentivar las mejoras en técnicas docentes, elaboración de materiales docentes on-line, etc.

Con la finalidad de apoyar las actividades e iniciativas relacionadas con la docencia que vayan en esta línea surgió la Plataforma de docencia en Economía. Las primeras Jornadas se celebraron en Granada con vocación de continuidad, y tuvieron como principal objetivo el intercambio de material docente entre los participantes. El material docente generado será puesto en breve a disposiciónde los docentes y será de enorme utilidad para nuestra propia docencia.

\section{Las primeras jornadas de docencia en Economía} Las primeras Jornadas de la Plataforma se celebraron entre el 15 y el 18 de abril en Granada. Las conferencias tuvieron lugar en el Hotel Abades Nevada Palace. Asimismo, con la finalidad de que sean unas verdaderas jornadas y haya una gran interacción entre los profesores participantes, la mayoría de los participantes se alojaron en el mismo hotel, el recomendado por la organización.

La celebración de las primeras Jornadas fue un éxito que superó las expectativas más optimistas, máxime si se tiene en cuenta la escasa difusión que tuvieron y la novedad del concepto. El número de participantes ascendió a 120 profesores procedentes de 32 universidades españolas. La distribución de los asistentes (Gráfico 1) permite observar la

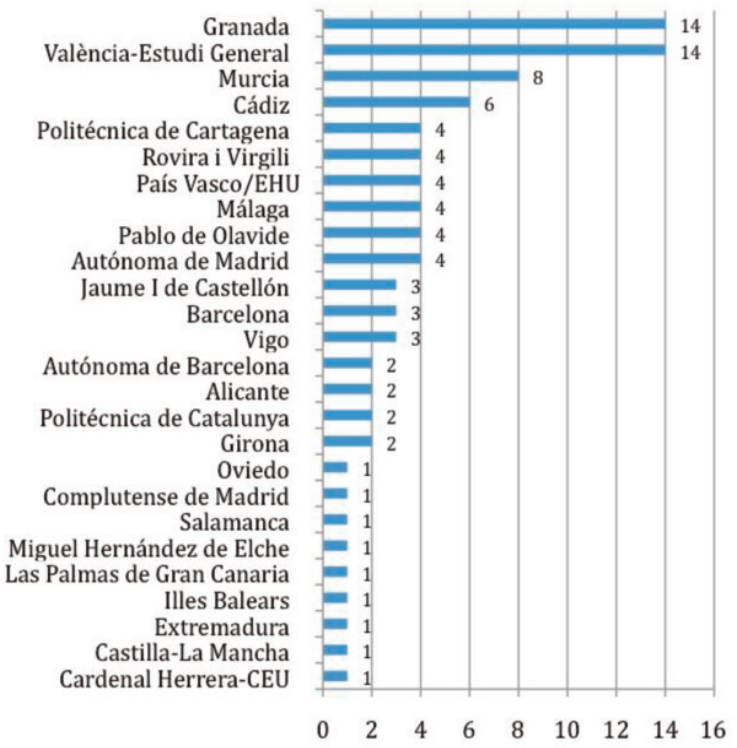

Gráfico 2. Número de ponencias presentadas por universidades 


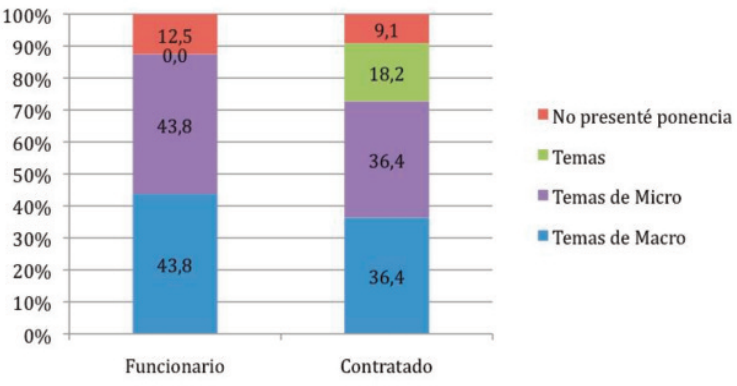

Gráfico 3. ¿En qué área(s) presentaste ponencias?

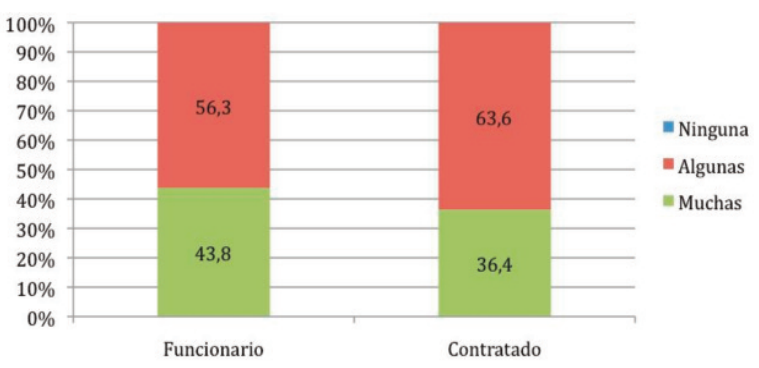

Gráfico 4. ¿Has obtenido alguna idea/material en las jornadas que piensas que puedes utilizar para mejorar tu docencia?

enorme diversidad de su procedencia, así como la intensa participación de profesores de ciertas universidades: la Universidad de Granada (16\%), de Valencia (9\%), Murcia (8\%) y Cádiz (7\%). Sólo estas cuatro universidades aglutinaban el $40 \%$ de los asistentes.

La participación en las Jornadas mediante ponencias fue también igualmente intensa. Se presentaron 92 ponencias en los tres bloques existentes: Microeconomía, Macroeconomía y Temas de Economía. El Gráfico 2 muestra el número de ponencias presentadas en función de la procedencia del ponente. De nuevo, los profesores de las anteriores cuatro universidades concentraron el $45,6 \%$ de las ponencias presentadas.

Con el ánimo de mejorar la calidad de futuras Jornadas, tras su finalización se animó a los participantes a que dieran su opinión sobre diversos aspectos de las mismas. A tal efecto se les envió un formulario en el que, además de la universidad de procedencia, sexo, edad, situación laboral, días de estancia en las jornadas, etc., se les preguntó si habían obtenido alguna idea o material aplicable para mejorar su docencia, también se les pidió su opinión sobre la realización regular de este tipo de iniciativas docentes, y una valoración sobre estas Jornadas en concreto (su organización, etc.).

La encuesta fue respondida por alrededor de un tercio de los asistentes (27 profesores), un 59,2\% de los cuales fueron mujeres. El 92,5\% de los encuestados permanecieron durante los dos días de duración de las Jornadas; y el 74,1\% tenían edades comprendidas entre los 36 y 45 años, y un 24,9\% de más de 45 años. Respecto a la situación laboral un $51,8 \%$ de los encuestados eran funcionarios (titulares o catedráticos), mientras el resto $(48,1 \%)$ eran contratados.

Cómo se observa en el Gráfico 3, la mayor parte de los encuestados presentaron ponencias. En el caso de los profesores funcionarios un $12,5 \%$ de los asistentes no presen- taron ponencia, frente a un 9,1\% de los profesores contratados. En cuanto a la preferencia de los temas elegidos, destaca que, tanto en el caso de los profesores funcionarios como de los profesores contratados, presentaron el mismo número de ponencias en micro que en macro. Destacando que los profesores contratados fueron los únicos ponentes en el bloque de Temas.

En lo referente a la utilidad en sí de las Jornadas como método de intercambio de experiencias, ideas o materiales, el Gráfico 4 muestra como el 100\% de los encuestados declaran que han obtenido algunas o muchas ideas para mejorar su docencia. Este gráfico muestra que no existen diferencias significativas entre funcionarios y personal contratado. Así, un 43,8 y un $36,4 \%$ de los funcionarios y profesores contratados encuestados declaran haber obtenido muchas ideas.

La valoración de este tipo de Jornadas por parte de los profesores encuestados fue igualmente positiva. Así, el Gráfico 5 muestra cómo el 93,8\% de los profesores funcionarios y el $98,9 \%$ de los contratados están muy a favor de la celebración de este tipo de iniciativas.

Finalmente, el Gráfico 6 muestra la valoración general de los profesores encuestados, tanto en relación a este tipo

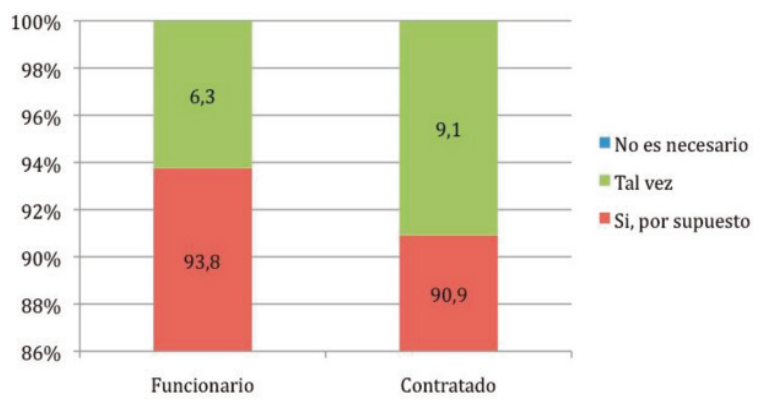

Gráfico 5. ¿Cuál es tu grado de valoración de este tipo de iniciativas docentes?

a) Funcionarios
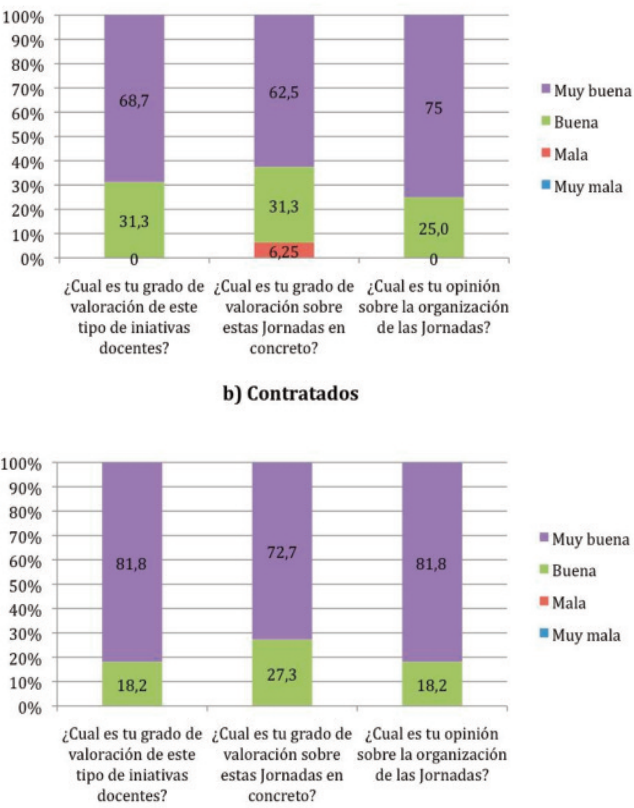

Gráfico 6. Valoración general de las Jornadas 
de iniciativas, como sobre estas Jornadas en concreto. La valoración general es muy positiva, existiendo, no obstante, algunas diferencias dignas de mención entre profesores funcionarios y contratados. Así, mientras que más de 8 de cada diez $(81,8 \%)$ profesores contratados valoran como muy buena este tipo de iniciativas, en el caso de los funcionarios este porcentaje se reduce al 68,7\%. Algo similar sucede en el caso de la valoración general de estas Jornadas, en las que el $72,7 \%$ de los contratados las valora como muy buenas, frente al 62,5\% de los funcionarios. Similarmente, los aspectos organizativos fueron muy bien valorados por el $81,8 \%$ de los profesores contratados, frente al $75 \%$ de los funcionarios.

Finalmente, la encuesta incluía un campo abierto en el que los profesores encuestados podrían hacer todo tipo de comentarios o manifestaciones. Muchos de ellos eran de agradecimiento por los esfuerzos realizados, y algunos otros eran tremendamente constructivos. Entre estos últimos podemos destacar algunas sugerencias, como por ejemplo, acotar el número de ponencias por autor, con el fin de evitar la duplicidad de presentaciones con la misma innovación docente. Y también, algunos de los encuestados han propuesto invitar a conferenciantes (preferiblemente internacionales) de acreditado prestigio en el ámbito de la innovación docente.

\section{Las próximas jornadas de docencia en Economía} En Valencia, con el apoyo de la Facultad de Economía y del Vicerectorat de Convergència Europea i Qualitat de la Universitat de València, los miembros de la Plataforma de Docencia en Economía se disponen a organizar las segundas Jornadas de Docencia. Disponiendo de los datos, la información sobre los aciertos y sobre todo los errores de la pasada Jornada, nos encontramos en el proceso de la preparación del encuentro, que en esta ocasión será internacional. El objetivo de desarrollar un marco donde nuestro trabajo tenga la máxima difusión será alcanzado de nuevo en Valencia.

\section{Referencias bibliográficas}

Michavila Pitarch, Francisco; Pérez García, Francisco; (Dir.) (2007). Análisis de las estrategias de adaptación de la oferta educativa de la Universidad de Alicante al Espacio Europeo de Educación Superior. Alcoy: Marfil, 2007.

Pastor, J.M. y F. Pérez (2009). La contribución socioeconómica de las universidades públicas valencianas. Publicacions de la Universitat de València, Servei de Publicacions de la Universitat de València.

Pérez, Francisco; Pastor, José Manuel; Hernández Pérez, Laura (2006). Diseño de la oferta futura de la formación superior de la Universitat de València. Valencia: Servei de Publicacions de la Universitat de València, 2006. 